\title{
Boston à l'heure française : religion, culture et commerce à l'époque des révolutions atlantiques
}

Boston's French Moment: Religion, Culture, and Commerce in the Age of Atlantic Revolutions

Mark Peterson

\section{(2) OpenEdition}

Journals

\section{Édition électronique}

URL : https://journals.openedition.org/ahrf/11925

DOI : 10.4000/ahrf.11925

ISSN : 1952-403X

Éditeur :

Armand Colin, Société des études robespierristes

Édition imprimée

Date de publication : 1 mars 2011

Pagination : 7-31

ISBN : 978-2-200-92677-9

ISSN : 0003-4436

\section{Référence électronique}

Mark Peterson, « Boston à l'heure française : religion, culture et commerce à l'époque des révolutions atlantiques », Annales historiques de la Révolution française [En ligne], 363 | janvier-mars 2011, mis en ligne le 01 mars 2014, consulté le 23 avril 2022. URL : http://journals.openedition.org/ahrf/11925 ; DOl : https://doi.org/10.4000/ahrf.11925 


\title{
BOSTON À L'HEURE FRANÇAISE : RELIGION, CULTURE ET COMMERCE À L'ÉPOQUE DES RÉVOLUTIONS ATLANTIQUES
}

Mark PETERSON

\begin{abstract}
À leur naissance, les jeunes États-Unis sont loin de rassembler une nation unifiée. Les villes et les États - tels Boston et le Massachusetts suivent fréquemment leurs intérêts propres et sont parfois en désaccord avec d'autres membres de l'union américaine. Cette diversité transparaît nettement, à l'époque révolutionnaire, de l'histoire des rapports entre Boston et la France, jadis perçue comme un ennemi acharné de cette ville très protestante. En explorant la manière dont des Bostoniens ont embrassé la culture et la religion française, cet article examine ainsi les conversions catholiques de quelques jeunes Bostoniens, puis les efforts d'une grande famille commerçante de Boston pour tirer profit des événements révolutionnaires en France et en Haïti.
\end{abstract}

Mots-clés : Boston, Nouvelle-Angleterre, commerce, religion, Haïti/ Saint-Domingue, Révolution américaine.

« Les affaires du peuple d'Amérique ont trop souvent été considérées comme un tout ». Ainsi s'exprime Benjamin Franklin, dans sa déposition devant la Chambre des communes en 1766, alors qu'il s'employait à justifier les résistances coloniales à l'acte du timbre. Pour Franklin, les émeutes qui éclataient dans de nombreuses villes portuaires étaient bien différentes des protestations mesurées des assemblées coloniales. Mais en tant que représentant officiel à Whitehall du Massachusetts, du New Jersey, de la Pennsylvanie et de la Georgie, soit des colonies aux caractéristiques et aux intérêts distinctivement différents, Franklin savait parfaitement que considérer le peuple largement disparate d'Amérique « comme un tout » 
était une erreur. Les intérêts qui avaient entraîné une colonie dans la rébellion ouverte étaient souvent bien différents de ceux qui avaient pu en motiver une autre ${ }^{1}$. Plus de cinquante ans plus tard, John Adams, un collègue de Franklin, soulignait cette extrême diversité parmi les colonies américaines lorsqu'il décrivait la Déclaration d'Indépendance, dont Franklin et lui-même avaient été les promoteurs au Congrès continental, comme presque miraculeuse : "Treize horloges sonnant ensemble en un mécanisme parfait qu'aucun artiste n'avait jusqu'alors réalisé $»^{2}$. Mais dans sa vision rétrospective, Adams oubliait ce qu'il avait fallu de contrainte et de persuasion afin d'accorder toutes ces « horloges », certaines, comme le Massachusetts et la Virginie, avaient nécessité d'être retardées, alors que d'autres, comme la Caroline du Sud ou la Pennsylvanie, avançaient plus vite que ce qu'elles souhaitaient. Adams ignorait aussi que certaines horloges ne s'étaient jamais déclenchées : les colonies de la Nouvelle Écosse, du Québec ainsi que l'est et l'ouest de la Floride qui avaient résisté à toutes les prières de rejoindre les «treize », tout comme la chaîne des colonies des Caraïbes, trop vulnérables, à la fois aux révoltes des esclaves et aux concurrents de l'empire, pour se passer de la protection de l'armée royale et de la $\mathrm{Navy}^{3}$. L'historiographie de la Révolution américaine a eu tendance à suivre la mémoire erronée et sélective d'Adams plutôt que de tenir compte de l'avertissement de Franklin. La plupart des efforts afin d'expliquer les causes de la Révolution américaine se sont concentrés sur les motifs, cachés ou manifestes (économiques idéologiques, constitutionnels ou culturels), partagés par les « treize », permettant ainsi d'expliquer la miraculeuse simultanéité de leur révolte commune ${ }^{4}$. De même, les historiens du début de la République des États-Unis supposent une uniformité et une solidité de la nation, comme si la diversité et la désunion des colo-

(1) The Examination of Doctor Benjamin Franklin, Relating to the Repeal of the Stamp Act, Philadelphie, 1766, p. 11.

(2) John Adams à Hezekiah Niles, le 13 février 1818, dans Charles Francis Adams (éd.), The Works of John Adams, Boston, 1852-1865, 10 volumes, vol. 10, p. 283.

(3) Andrew O'Shaughnessy, An Empire Divided: The American Revolution and the British Caribbean, Philadelphie, 2000.

(4) Sur des travaux généraux cherchant à présenter les causes de la Révolution, voir : Bernard BAILYN, The Ideological Origins of the American Revolution, Cambridge, 1967; T. H. BREEN, The Marketplace of Revolution: How Consumer Politics Shaped American Independence, New York, 2004 ; Nicole Eustace, Passion is the Gale : Emotion, Power, and the Coming of the American Revolution, Chapel Hill, 2008; Pauline MAIER, From Resistance to Revolution : Colonial Radicals and the Development of American Opposition to Britain, 1765-1776, New York, 1972; Robert MiddekAufF, The Glorious Cause : The American Revolution, 1763-1789, New York, 1982; Gordon Wood, The Radicalism of the American Revolution, New York, 1992. 
nies, soulignées par Adams, avaient subitement disparu de la Déclaration de Philadelphie ${ }^{5}$. Cette tendance est particulièrement éclatante dans les histoires des relations nord-américaines avec le monde lors de la séquence révolutionnaire. Il est bien plus aisé d'imaginer une « Amérique » unifiée, ou d'envisager les conflits qui régnaient au sein même des États-Unis sur la question des affaires étrangères comme une simple opposition entre partis politiques nationaux, plutôt que de rappeler que les États indépendants conservaient leur spécificité, poursuivaient leurs intérêts propres et envisageaient de remettre en question l'union nationale bien après l'épisode de la guerre de Sécession.

À l'opposé de cette tendance dominante, cet article prend le parti de Franklin. Il suppose que « les affaires du peuple d'Amérique ont trop souvent été considérées comme un tout » et s'intéresse à ce que l'on peut obtenir si on les considère séparément. Plus précisément, il s'attache prioritairement à la ville de Boston, le centre originel de la rébellion contre le Parlement et la Couronne, cette horloge qui devait sonner la première et de manière la plus intense. Lorsque Franklin et Adams imploraient le Congrès continental de déclarer l'Indépendance, la question fut de savoir si les colonies les plus hésitantes accepteraient que « le parti de Boston était le parti de toute l'Amérique ». En 1774, le Parlement avait puni Boston pour la destruction du thé de l'East India Company. Les actes coercitifs avaient fermé le port, suspendu le gouvernement local et placé la colonie sous la férule militaire. En soutien de sa capitale, le Massachusetts était en guerre ouverte avec les troupes de sa majesté depuis avril 1775, son gouvernement ad hoc agissant comme un État indépendant pendant plus d'une année avant que les douze autres colonies ne le rejoignent et concluent mutuellement "un solide accord d'amitié $»^{6}$. Mais dans leur besoin désespéré de soutien afin de contrer la force militaire du roi George, certains, à Boston, commencèrent à se demander si le parti de «toute l'Amérique » allait demeurer indéfiniment celui de Boston... Quelques ultra-nationalistes, comme John Adams, qui avait joué un rôle déterminant au sein du Congrès continental, étaient convaincus que Boston allait servir de guide à la nation en devenir. D'autres, envisageaient les nouveaux États-Unis avec beaucoup plus de circonspection lors de ses deux premières décennies.

(5) Joyce Appleby, Inheriting the Revolution, Cambridge, 2000; Stanley Elkins et Eric McKitrick, The Age of Federalism, New York, 1993 ; Gordon S. Wood, Empire of Liberty : A History of the Early Republic, 1789-1815, New York, 2009.

(6) Articles of Confederation of the United States of America. 
Le danger d'une nouvelle alliance avec d'anciennes colonies dont « les coutumes, les manières et les habitudes sont si peu semblables, et leurs relations si rares $\gg^{7}$ semblait un problème crucial particulièrement pour Boston, la ville étant depuis toujours exceptionnellement indépendante. Le reste de l'Amérique britannique possédait une structure coloniale économique marquée, forgée par une philosophie mercantiliste inspirée par les actes de navigation du Parlement. La plupart des autres colonies produisaient des matières premières très recherchées en Grande-Bretagne (sucre, tabac, riz, indigo), importaient des produits manufacturés britanniques en retour et prospéraient à l'intérieur de ce système fermé sous la direction de gouverneurs nommés par la couronne et la protection de la Royal Navy ${ }^{8}$. Ce ne fut jamais le cas de Boston. La Nouvelle-Angleterre, son arrière-pays, ne produisait pas de cultures de rapport et ses marchands commerçaient à l'intérieur d'un marché atlantique aux larges dimensions, souvent en dehors des frontières de l'autorité britannique, le tout formant une économie complexe qui ressemblait à celle de la métropole, tout en entrant en compétition (plus qu'elle n'en dépendait) avec elle9. Même lorsque le Massachusetts fut devenu une province royale en 1691, et bien plus fréquemment que dans les autres colonies, Boston avait réussi à persuader la couronne de nommer comme gouverneurs des hommes issus de son sol, maintenant ainsi de profondes racines d'auto-gouvernance et un sens de l'autonomie politique qui s'identifiait à son indépendance économique $^{10}$. En termes de culture, Boston fut fondée comme un paradis pour les puritains dissidents aux normes anglicanes, et elle conservait cet esprit de résistance de manière beaucoup plus intense que dans n'importe quel autre lieu de l'Amérique britannique. Les soldats britanniques envoyés pour occuper Boston en 1768 furent étonnés du caractère puritain de la ville (fermée à toute circulation, commerce et jeux le dimanche), comme ils le furent de sa résistance aux lois fiscales du Parlement ${ }^{11}$. Ses traditions culturelles, politiques et commerciales furent la source de la rébellion

(7) Adams à Niles, Works of John Adams, op. cit., vol. 10, p. 283.

(8) John J. McCusker et Russell R. Menard, The Economy of British America, 1607-1789, Chapel Hill, 1991.

(9) McCusker et Menard, op. cit., p. 91-117; Bernard Bailyn, The New England Merchants in the Seventeenth Century Cambridge, 1955; Stephen InNEs, Creating the Commonwealth: The Economic Culture of Puritan New England, New York, 1995; Margaret E. Newell, From Dependency to Independence : Economic Revolution in Colonial New England, Ithaca, 1999.

(10) Leonard Woods Labaree, Royal Government in America : A Study of the British Colonial System before 1783, New York, 1958.

(11) Oliver Morton Dickerson, Boston Under Military Rule : As Revealed in A Journal of the Times, Boston, 1936. 
contre la métropole, mais provoquaient également des réserves quant à son association avec des colonies inconnues façonnées à l'image de la Grande-Bretagne.

Notre article ne s'attache pas à évoquer les nationalistes bostoniens revendiqués comme Adams (parmi d'autres peu nombreux), mais plutôt tous ceux qui, à Boston, adhéraient à une longue tradition revendiquant l'indépendance de l'État et de la ville comme un moyen de protéger ses intérêts, de préserver sa propre identité culturelle et de mener une politique extérieure personnelle au sein d'un vaste monde atlantique. Les relations de la ville avec la France à cette période offrent donc un terrain idéal afin d'apprécier ces efforts, car les transformations à l'œuvre, tout d'abord en raison de l'attitude de Boston vis-à-vis de la France dans les années 1770 et 1780, puis au sein du monde politique, économique et culturel français et colonial après 1789 , étaient si radicales et dramatiques que la voie de l'indépendance suivie par Boston par rapport au reste des États-Unis est tout à fait éloquente. Afin de rendre ces généralités explicites, nous suivrons la carrière d'un petit groupe de Bostoniens ayant saisi les opportunités offertes par les transformations brutales des relations entre la ville et l'empire français, et qui ont ensuite refondé la culture et le commerce de la ville. Nous commencerons avec Samuel Breck et John Thayer, les premiers natifs de Boston à avoir reçu une éducation en France, à avoir été convertis au catholicisme romain et enfin à avoir introduit leurs pratiques confessionnelles dans la ville, des transformations rendues possibles par l'ouverture culturelle produite à la suite de l'alliance franco-américaine de 1778. Puis, nous nous attacherons à l'attitude commerciale profitable et calculatrice visà-vis du républicanisme français adoptée par Thomas Handasyd Perkins, sa famille et ses associés. Les affaires de la maison Perkins à Saint-Domingue furent ainsi fondées sur une estimation perpétuelle du contexte et des évolutions des perspectives commerciales, mais aussi sur la liberté des échanges et la/l'(in)stabilité politique du monde atlantique révolutionnaire français. En suivant ces acteurs immergés dans les affaires françaises, il apparaît clairement que leurs réponses originales aux transformations des relations avec la France prennent racine dans les anciennes traditions de la ville de Boston et ne peuvent pas être facilement confondues avec une réponse américaine globale à la Révolution française.

La perspective d'un débarquement de la marine française sur les côtes du Massachusetts fut très longtemps le pire cauchemar de Boston. 
Afin de se protéger de décennies de conflits entre la France et la GrandeBretagne, la ville a maintenu plusieurs forteresses, une sur Island Castle, située directement en face du port de Long Wharf, le centre du négoce de la ville, et une sur Fort Hill, dans l'ancienne partie sud de la ville ${ }^{12}$. Mais les attaques ne vinrent jamais. La seule flotte à s'être emparée du port de Boston fut britannique, durant l'occupation militaire de la ville entre 1768 et 1776 . Et juste après que Boston et ses alliés de la Nouvelle-Angleterre eurent repoussé la Royal Navy du roi George hors de la ville, la nouvelle alliance entre le roi Louis et les États-Unis permit à la marine française d'entrer à Boston dans des conditions largement inattendues.

De toutes les colonies britanniques en Amérique, Boston et la Nouvelle-Angleterre participèrent intensément aux guerres contre la France, et ce dès 1689, lorsque le nouveau monarque britannique, Guillaume III, mit en place une alliance contre Louis XIV. Les conflits se déplacèrent vers le nord de l'Amérique et la proximité de la NouvelleAngleterre et de la Nouvelle-France se traduisit par des guérillas brutales de chaque côté des forêts du Nord. Pendant presqu'un siècle, les habitants de la Nouvelle-Angleterre avaient appris à croire que les catholiques français étaient leurs ennemis invétérés. Les pierres tombales au $\mathrm{XVIII}^{\mathrm{e}}$ siècle dans la campagne du Massachussetts racontent l'histoire de soldats qui «battent, intrépides, les campagnes / sans jamais capituler devant l'ennemi gaulois $\rangle^{13}$. Entre 1754 et 1761 , un tiers de la population masculine adulte participa à la guerre de Sept Ans contre les « ennemis gaulois $\gg{ }^{14}$. Dans la ville de Boston, chaque 5 novembre, se tenait ce que l'on appelait la "fête du Pape », soit l'anniversaire de l'échec de Guy Fawkes dans sa tentative de faire sauter le Parlement en 1605. Des gangs des rues s'affrontaient afin de prendre possession d'une effigie du pape exhibée dans toute la ville sur un chariot. Aux vainqueurs revenait l'honneur de brûler l'effigie dans un feu de joie ${ }^{15}$. Bien que les monarques

(12) T. W. Higginson, « French and Indian Wars », et E. L. Bynner, « Topography and Landmarks of the Provincial Period », dans Justin Winsor, (éd.), The Memorial History of Boston, 4 volumes, Boston, 1886, vol. 2, p. 93-130 et p. 491-532; Walter Muir WhitehiLL et Lawrence W. Kennedy, Boston : A Topographical History, $3^{\mathrm{e}}$ edition, Cambridge, 2000.

(13) Mark A. Peterson, «Stone Witnesses, Dumb Pictures, and Voices from the Grave : Monuments and Memory in Revolutionary Boston ", à paraître dans David GoBel et Daves RosselL (éd.), Commemoration and the American City, Charlottesville, 2010.

(14) Fred Anderson, A People's Army: Massachusetts Soldiers and Society in the Seven Years'War, Chapel Hill, 1984, p. 3-25.

(15) Voir Peter Benes, « Night Processions : Celebrating the Gunpowder Plot in England and New England », dans New England Celebrates : Spectacle, Commemoration, and Festivity, Boston, 2001 ; Samuel Eliot Morison, Harrison Gray Otis, 1765-1848 : The Urbane Federalist, Boston, 
Bourbons français fussent tout au long du XVIII ${ }^{\mathrm{e}}$ siècle souvent en désaccord avec le Vatican, dans les mentalités populaires de Boston, les deux personnages, le pape et le roi français, ne faisaient qu'un: l'unique modèle de la tyrannie absolutiste, temporelle et spirituelle, et tous les Français étaient leurs sujets serviles. Les préjugés étaient si forts que lors de la crise impériale des années 1760, beaucoup d'habitants de la Nouvelle-Angleterre étaient persuadés que les nouveaux impôts et les décrets nuisibles du Parlement devaient être le résultat des efforts secrets des Jésuites et des Français ${ }^{16}$.

Le traité d'alliance de 1778 entre la France et les États-Unis allait opérer des changements radicaux dans les relations entre Boston et tout ce qui était de près ou de loin d'origine française. Le siège victorieux de Boston ayant forcé les Anglais à abandonner la ville, Boston devint le port nord américain le plus accessible pour la marine française pendant les premières années de la guerre. La flotte française devint une présence familière du port de Boston dès l'été 1778, où elle pouvait être en toute sécurité réparée et abritée. En avril de la même année, le comte d'Estaing quitta Toulon à la tête d'une flotte de 26 bateaux. Une violente tempête contrecarra ses plans d'attaque des Britanniques au large de Newport dans le Rhode Island, et l'obligea à rejoindre Boston afin de réparer ses navires endommagés. Et là, selon les souvenirs de Samuel Breck ${ }^{17}$, la foule pressée aux pontons fut interloquée de découvrir que les marins français n'étaient pas « une troupe de soupe-maigre décharnés et affamés » à laquelle elle s'attendait et doutait même que ces "robustes individus puissent appartenir à la race des "mounseers" aux joues creuses et aux jambes de faucheux ». Lorsque Nathaniel Tracy, un riche marchand de Boston, invita à dîner l'amiral d'Estaing et Philipp Joseph de l'Etombe, le consul français, il se donna beaucoup de mal afin de servir à ses invités une soupe aux grenouilles. M. de l'Etombe, à son grand étonnement, extirpa une grenouille de son assiette et s'exclama : «Ah, mon Dieu! une grenouille! Puis se tournant vers le gentilhomme assis à ses côtés, il lui donna l'animal. Ce dernier la fit alors circuler tout autour de la table et elle finit sa course dans l'assiette de l'amiral ${ }^{18}$.

1969, p. 7-8; Alfred F. Young, Liberty Tree: Ordinary People and the American Revolution, New York, 2006, p. 111.

(16) BaiLyn, Ideological Origins, op. cit., p. 63-66, 98 et 144-158.

(17) Il exista deux Samuel Brecks, le père et le fils. Les mémoires utilisés ici sont l'œuvre du fils, Samuel Breck le jeune. Horace ScudDER (éd.), The Recollections of Samuel Breck, Philadelphia, 1877.

(18) Ibid., p. 24-27. 
Pourtant, malgré la foi inébranlable de Nathaniel Tracy en la soupe aux grenouilles, selon lui le " plat national français », il existait de nombreux éléments dans les relations passées entre la France et Boston qui remettaient en cause ce type de stéréotypes. L'un était la présence des réfugiés huguenots français à Boston dont certaines familles, comme les Faneuil, les Bowdoin et les Revere s'étaient tellement bien fondues dans la culture dominante de Boston qu'elles avaient fini par obtenir des positions dominantes dans les mondes du négoce, de l'artisanat ou de la politique. L'église protestante française qu'ils avaient fondée sur School Street devait même se maintenir pendant plusieurs décennies jusqu'au milieu du $\mathrm{XVIII}^{\mathrm{e}}$ siècle, lorsqu'elle finit par se mêler aux institutions puritaines ${ }^{19}$. Un autre de ces éléments était l'existence d'anciennes et profitables relations existant entre la communauté des marchands de Boston et les colons français d'Acadie, connus sous le nom de " Français neutres », qui devaient d'ailleurs rester dans la région bien après que la Grande-Bretagne eut repris officiellement possession de la Nouvelle Écosse en 1710. Malgré leur catholicisme, les Acadiens étaient d'excellents clients des marchands de la côte de Boston jusqu'à ce que l'armée britannique les en expulse dans les années $1750^{20}$. Et donc, malgré de puissants préjugés forgés par des décennies de guerre, il était néanmoins possible pour les Bostoniens d'imaginer que les Français n'étaient pas essentiellement, et de manière permanente, leurs ennemis naturels, mais qu'ils étaient simplement les victimes de tyrans spirituels et temporels : les papes et les rois qui les dirigeaient. À partir de ce nouvel éclairage, le soutien proclamé de la France à la cause révolutionnaire de Boston permettait de laisser penser que le changement était possible.

Lors de la guerre d'Indépendance américaine, le commerce fut le vecteur de ce changement, en particulier dans les attitudes culturelles de Boston envers tout ce qui était français. La présence française offrit aux marchands locaux la possibilité de fournir aux troupes de la nourriture, des vêtements, des fournitures navales et des services financiers. En 1779, Samuel Breck l'aîné se vit offrir, par le gouvernement de Louis XVI, la fonction d'agent permanent auprès des troupes françaises de Boston, ce

(19) Charles C. Smith, «The French Protestants in Boston», dans Winsor, (éd.), The Memorial History of Boston, op. cit., vol. 2, p. 249-268.

(20) George Rawlyk, Nova Scotia's Massachusetts : A Study of Massachusetts-Nova Scotia Relations, 1630-1784, Montreal, 1973; Geoffrey PlanK, An Unsettled Conquest : The British Campaign Against the Peoples of Acadia, Philadelphia, 2001; John Mack FAragher, A Great and Noble Scheme: The Tragic Story of the Expulsion of the French Acadians from their American Homeland, New York, 2005. 
qui voulait dire qu'il « vendait leur marchandise, négociait leurs effets de change et aménageait leurs bateaux de guerre avec tout ce qu'ils désiraient ${ }^{21}$. Ce type de dispositions avait de très anciens précédents dans l'histoire de Boston et quelques-unes des grandes fortunes de la ville au XVIII ${ }^{\mathrm{e}}$ siècle s'étaient construites grâce au ravitaillement de la flotte militaire britannique à partir de $1689^{22}$. Mais ces relations commerciales débouchaient également sur des rencontres sociales et culturelles. $\mathrm{Au}$ début $\mathrm{du} \mathrm{XVIII}^{\mathrm{e}}$ siècle, les guerres avaient permis à Boston de s'aventurer sur d'autres routes qui l'avaient menée en Angleterre, aux ProvincesUnies et en Allemagne ${ }^{23}$. Et à présent la France devenait un autre segment du monde atlantique bostonien, de manière à la fois nouvelle mais également familière.

La famille Breck fut obligée de quitter Boston lors de l'occupation britannique en 1775-1776, mais à son retour, à la suite du départ des Britanniques, Samuel Breck l'aîné acquit une belle propriété située en face du parc de Boston. Dans ces murs, il organisa une fête gigantesque pour la ville entière en l'honneur de la naissance du Dauphin, Louis Joseph, en 1781, une première à Boston ${ }^{24}$. Cette cérémonie en l'honneur du Dauphin n'est qu'un des nombreux éléments attestant de l'engouement pour tout ce qui était français et qui affecta la ville pendant toute la guerre. Ainsi, si quelques Bostoniens s'étaient initiés au français avant 1778, la demande de tuteurs pour apprendre la langue explosa après cette date. Breck l'aîné vit ses affaires avec les Français entravées par ses difficultés à s'exprimer dans cette langue et, devant s'en remettre au consul français l'Etombe pour les traductions, il fut bientôt obligé de partager avec lui ses profits. Breck et John Hancock louèrent les services d'un enseignant afin d'apprendre le français, apparemment sans grand succès. Mais il déplaça ses ambitions vers ses enfants, déterminé à ce qu'ils apprennent la langue de Voltaire, même si lui-même en avait été incapable ${ }^{25}$.

En 1782, lorsque Breck l'aîné envisagea d'envoyer en France son fils, Samuel Breck, alors âgé de 11 ans, afin d'y être éduqué (une autre première dans l'histoire de Boston) et alors que la guerre faisait encore

(21) Recollections of Samuel Breck, op. cit., p. 43; Robert A. EAST, Business Enterprise in the American Revolutionary Era, New York, 1938, p. 57-58.

(22) Michael Batinski, Jonathan Belcher, Colonial Governor, Lexington, Kentucky, 1996.

(23) Mark A. Peterson, « Theopolis Americana: The City-State of Boston, The Republic of Letters, and the Protestant International, 1689-1739 », dans Bernard BAILYN (éd.), Soundings in Atlantic History : Latent Structures and Intellectual Currents, 1500-1825, Cambridge, 2009, p. 329-371.

(24) Recollections of Samuel Breck, op. cit., p. 38.

(25) Ibid., p. 46-48. 
rage, il se tourna vers Louis-Philippe de Rigaud, marquis de Vaudreuil, commandant en chef des forces navales en Amérique, et résident de Boston $^{26}$. Ce dernier lui conseilla le collège de Sorèze, près de son village de Vaudreuil, dans le Bas Languedoc, un établissement dirigé par des moines bénédictins qui combinait enseignement militaire et littéraire. Lors de son voyage, le jeune Breck apprit, avec plaisir, qu'il existait en France un engouement pour Boston comparable à celui que sa ville vouait à la France. Dans la mesure où la guerre d'Indépendance y avait débuté et parce que les Européens n'avaient que des notions géographiques limitées sur l'Amérique, Boston était devenue pour tous « l'Amérique révolutionnaire ». À Nantes, le jeune homme assista pour la première fois à une pièce de théâtre (un genre interdit dans sa ville de Boston) et les actrices devaient bientôt le surnommer « le petit Bostonien », car « c'était alors le nom réservé à tous les Américains ». Il apprit également une chanson française, alors à la mode, dont le refrain disait : «Bon, Bon, Bon, / C'est à Boston / Qu'on entend souflé (sic) les canons » et découvrit un jeu de carte, lui aussi dénommé Boston, jeu qui devait ensuite rester en vogue dans toute l'Europe (sauf en Grande-Bretagne) pendant plusieurs dizaines d'années ${ }^{27}$. Par la suite, lors de son long périple qui le conduisit à La Rochelle, Rochefort, Bordeaux et Toulouse, Breck fils évoque, également, un café de Montauban baptisé le « Café de Boston $»^{28}$.

Breck fils devait passer quatre années au collège de Sorèze et, sous la conduite des moines bénédictins, il se convertit au catholicisme romain, malgré son éducation et son ascendance puritaine. Un peu plus tôt dans le siècle, d'autres jeunes voyageurs venus de Boston en Europe avaient rencontré des ecclésiastiques catholiques, de manière suffisamment fréquente pour que des formes modélisées de réactions se développent, tout au moins à travers les témoignages écrits rédigés à la suite de ces rencontres. Le modèle classique mettait en scène un jeune voyageur et son interlocuteur catholique (souvent un Jésuite) dans une conversation animée autour de la doctrine religieuse, le jeune voyageur surpassant son adversaire grâce à sa logique implacable et sa profonde foi protestante ${ }^{29}$.

(26) George Washington à Louis Philippe de Rigaud, Marquis de Vaudreuil, pariers George Washington Papers à la Bibliothèque du Congrès, 1741-1799 : Séries 3d Varick Transcripts, Soussérie D, Foreign Officers and Subjects of Foreign Nations, Letterbook 2, http://memory.loc.gov/cgibin/ampage? collId $=$ mgw3 \&fileName $=$ mgw3d/gwpage002 $. \mathrm{db} \& \mathrm{recNum}=0$

(27) Dans Guerre et Paix de Tolstoï, le comte Rostov joue fréquemment au Boston. Voir le livre 1, chapitre 18.

(28) Recollections of Samuel Breck, op. cit., p. 53-58.

(29) Peterson, « Theopolis Americana », op. cit., p. 339, et note 35, p. 578. 
L'expérience du jeune Samuel Breck fut sensiblement différente et cet écart peut s'expliquer par le changement d'attitude de Boston envers la France pendant sa jeunesse. À sa grande surprise, les moines, explique Samuel, «m'ont toujours témoigné une tendresse paternelle et ont porté une attention particulière à mon bonheur, présent et à venir », mais ils n'ont jamais fait « le moindre effort afin de m'éloigner de la religion de mes ancêtres », un point sur lequel son père avait bien insisté. D'ailleurs, sa conversion provenait de l'influence d'un de ses condisciples, « un jeune homme d'une grande et rigoureuse piété » dont le comportement « studieux, ordonné, exemplaire à tous les égards » s'offrit en modèle au jeune Breck et fut le facteur déterminant de sa conversion, le moine supérieur insistant d'ailleurs pour que Breck prenne une semaine de réflexion avant de l'envoyer se confesser.

Si la conversion de Breck peut être attribuée à son éducation et à sa culture, et pas uniquement à son passage au collège de Sorèze, elle a pu également être influencée par les transformations qui avaient touché Boston et qui avaient permis aux descendants des puritains de reconnaître une part essentielle d'humanité dans le cœur de leurs anciens ennemis ${ }^{30}$. Peut-être même pourrions-nous qualifier la conversion de Breck à la religion catholique de « conversion sociale »? Il n'est d'ailleurs pas surprenant qu'à son retour à Boston, il ait embrassé une position médiane, située entre la religion calviniste de son enfance et son éducation catholique, rejoignant l'Église épiscopale aux lendemains de la Révolution. Jamais conduit par le fanatisme, Breck souhaitait se conformer à la culture et à la société dans laquelle il vivait, société qui, après 1778, avait alloué à Boston un espace de tolérance envers la France et sa religion ${ }^{31}$.

À peu près à la même époque, un autre jeune bostonien s'était converti, lui aussi, à la religion catholique, mais dans des circonstances bien différentes. John Thayer, comme Samuel Breck le jeune, était issu d'une vieille famille puritaine de Boston, mais il était plus âgé que Breck et avait déjà reçu une solide éducation avant de voyager en Europe. À l'âge de 16 ans, il fut envoyé au collège de Yale à New Haven. Lorsque l'élite bostonienne envoyait ses enfants dans le lointain Yale plutôt qu'à Harvard, pourtant proche, cela signifiait qu'elle préférait le calvinisme rigoureux de Yale à l'éducation latitudinaire d'Harvard, qui penchait d'ailleurs de plus en plus vers l'unitarisme. À son retour de Yale, fidèle

(30) Recollections of Samuel Breck, op. cit., p. 77-78.

(31) Ibid., p. 83. 
à lui-même, John Thayer devint «pasteur de la secte puritaine » et se vit confier plusieurs chaires vacantes autour de Boston; pendant la guerre, il devint même aumônier auprès de la milice du Massachusetts stationnée à Castle William dans le port de Boston. Mais pendant ce temps-là, Thayer entretenait « un penchant secret pour le voyage et décida de se rendre en Europe afin d'apprendre les langues les plus parlées, d'acquérir des connaissances sur la constitution des États, sur les mœurs, les coutumes, les lois et les gouvernements des principales nations $»^{32}$.

C'est en 1781, soit un an avant que Samuel Breck rejoigne l'Europe, que Thayer s'embarqua dans son projet de voyage, éclairé par les Lumières du temps. Il gagna d'abord la France, y séjourna pendant dix mois, étudiant la langue et la politique, sans afficher un quelconque intérêt pour une conversion religieuse. D'ailleurs, alors qu'il était tombé malade, il refusa que des prêtres catholiques s'approchent de lui, redoutant que profitant d'un moment de faiblesse physique, ils le pressent de se convertir. Il se rendit également en Angleterre, y resta plusieurs mois, et lorsqu'on lui demanda de prêcher devant des congrégations de dissidents, il se rendit compte, à son grand désarroi, que ces derniers étaient très éloignés de ses positions théologiques personnelles. Ce désaccord entre protestants l'affecta et le conduisit à ses premières réflexions sur « la nécessité des autorités temporelles de définir le véritable sens des Évangiles ». À son retour en France, il commença à ressentir « une impression plus favorable envers la religion catholique », il décida de visiter Rome, ville qui lui permettrait d'étudier la doctrine du catholicisme tout en poursuivant ses autres projets intellectuels. Et en Italie, ses préjugés s'effacèrent devant la gentillesse de ses hôtes : " une telle bonté, une telle cordialité envers les étrangers, un protestant qui plus est, m'a touché et surpris. Cette religion, dis-je, ne favorise pas la misanthropie, et n'inspire pas, comme on me le répétait, des sentiments d'aversion et d'intolérance ». Il fut également impressionné par la grandeur de la Rome antique et par la force indéniable de l'idée qui voulait « que Dieu, dans sa dignité, ait établi le centre de l'idolâtrie, le centre de la vraie foi, la première ville au monde et la capitale de son royaume[...] Cette idée me ravit, et j'étais admiratif de l'éloquence des prêtres en

(32) Of the Conversion of the Reverend John Thayer Hartford, Connecticut., 1832. Voir aussi : Thomas W. Jodziewicz, «American Catholic Apologetical Dissonance in the Early Republic? Father John Thayer and Bishop John Carroll », The Catholic Historical Review, vol. 84, n 3, juillet 1998, p. 455-476; Richard H. Clarke, «A Noted Pioneer Convert of New England: Rev. John Thayer, 1758-1815 », American Catholic Quarterly Review, vol. 29, 1904, p. 138-166; Percival MerritT, "Sketches of the Three Earliest Roman Catholic Priests in Boston », Colonial Society of Massachusetts Publications, vol. 25, Cambridge, 1923, p. 173-229. 
chaires, j'imaginais qu'il était alors possible de traiter la vérité de manière aussi sublime ». En bref, grâce à ses rencontres avec les monuments, la culture, grâce à ses fréquentations et à la gentillesse qu'on déployait à son égard dans le centre du catholicisme européen, Thayer constatait que ses goûts spirituels et esthétiques étaient en train d'évoluer ${ }^{33}$.

Il s'ensuivit une expérience de conversion, bien plus puissante que celle de Samuel Breck, et, peut-être, la plus « protestante » des conversions à la religion catholique. Et à bien des égards, le récit de Thayer se confond avec les étapes classiques de la «morphologie de la conversion ", qui rend compte de manière tout à fait caractéristique de l'expérience de la conversion puritaine ${ }^{34}$. Connaissant le caractère orthodoxe de son éducation, Thayer avait probablement identifié cette morphologie comme étant la seule structure convaincante qu'une conversion authentique devait revêtir, et il n'est pas alors surprenant qu'elle ait pris cette forme. Thayer, d'ailleurs, rend compte des débuts de son attirance intellectuelle et émotionnelle pour cette religion étrange, suivis par une sensation intime de résistance et d'incapacité à croire (« en un mot, mon esprit était convaincu, mais mon cœur restait inchangé $»)^{35}$. Cette résistance devait finalement être brisée par une combinaison de lectures de textes sacrés, d'échanges avec de vrais croyants et des guides spirituels (dans son cas des jésuites et des frères augustiniens), des prières ferventes, le tout contrebalancé par le doute, pour finalement donner, dans le langage habituel des puritains convertis, une rencontre providentielle avec des textes, des hommes et des événements qui transformèrent dramatiquement son cœur. Comme tout bon protestant l'affirmait, il s'agissait d'une conversion essentiellement passive (un acte de Dieu et non de soi) qu'il racontait par la voix passive du pêcheur abject et impuissant :

«[...] un petit livre intitulé Manifesto di un Cavalaiero Christiano convertito all Religione Catholica [...] fut placé entre mes mains [...] J'avais le secret pressentiment qu'il allait me donner le coup fatal [...] En outre, je ne résistais plus à la grâce divine comme auparavant : Dieu parlait à mon cœur et en même temps il éclairait mon esprit, me donnant la grâce qui me permettait de surmonter tous les obstacles qui m'avaient jusqu'alors retardé $»^{36}$.

(33) Conversion of the Reverend John Thayer, op. cit., p. 8-11.

(34) Edmund S. Morgan, Visible Saints : The History of a Puritan Idea, Ithaca, 1965, p. $66-73$.

(35) Conversion of the Reverend John Thayer, op. cit., p. 15.

(36) Ibid., p. 15-17. 
À l'exception de la référence à la Religione Catholica, le récit de la conversion de Thayer aurait pu être jugé valable devant n'importe quelle congrégation calviniste la plus traditionaliste de Boston, ou encore pour le cas qui nous occupe, de New Haven ${ }^{37}$. Ainsi transformé, Thayer retourna en France en 1783 afin d'y suivre le séminaire de Saint-Sulpice à Paris et fut ordonné prêtre quatre ans plus $\operatorname{tard}^{38}$. Toutefois, depuis le début, son intention ne fut pas de s'immerger dans le monde catholique français, mais de retourner à Boston en tant que missionnaire de sa nouvelle foi : « Je ne désire rien de plus; dans ce but je souhaite revenir dans mon pays d'origine, dans l'espoir, humblement, d'être l'instrument de la conversion de mes compatriotes $»{ }^{39}$. À travers sa propre expérience, John Thayer avait découvert ce qui était pour lui la seule et unique manière d'être un puritain dans le Boston post-révolutionnaire. Depuis de très nombreuses années, Boston représentait une ville à la fois œcuménique et réceptive à toutes les formes du protestantisme, du haut clergé anglican aux spiritualistes quakers en passant par le « vieux corps des unitariens » et tout le reste ${ }^{40}$. C'est uniquement en tant que catholique romain que quelqu'un comme John Thayer pouvait se permettre de continuer à être chrétien tout en affichant une croyance fervente et convaincue de sa légitimité selon laquelle il possédait la seule vraie foi et avait le devoir d'en instruire les autres afin de leur éviter d'être damnés pour l'éternité.

De retour à Boston en 1790, John Thayer, avec l'aide financière de Samuel Breck le jeune, fonda une congrégation catholique romaine dans l'immeuble situé sur School Street et qui était, des décennies plus tôt, le lieu où se retrouvaient les protestants français ${ }^{41}$. Pour Breck, un homme pacifique et œcuménique, ce bâtiment était un choix naturel, une marque de continuité avec l'histoire des Français dans la ville, et un moyen de suggérer que, comme les huguenots, les catholiques français étaient à présent un allié, un de plus parmi tous ceux que la ville comptait au sein du monde atlantique. Mais Thayer, à la différence de Breck, continua d'exercer sa prêtrise avec la vigueur d'un fanatique puritain. En 1785, bien avant son retour à Boston, il s'en prit à Paris à la foi de John et Abigail Adams, criti-

(37) Voir MarkA. Peterson, « Puritanism and Refinement in Early New England : Reflections on Communion Silver », William and Mary Quarterly, 3d ser., 58, avril 2001, p. 307-346.

(38) Jodziewicz, « American Catholic Apologetical ... », op. cit., p. 457.

(39) Conversion of the Reverend John Thayer, op. cit., p. 21.

(40) Perry Miller, (éd.), The Transcendentalists : Their Poetry and Prose, Garden City, NJ, 1957, p. IX.

(41) Recollections of Samuel Breck, op. cit., p. 83-84 et p. 116-117. 
quant Calvin et les autres réformistes ${ }^{42}$. De manière très puritaine, Thayer fut prompt à résister à l'autorité épiscopale de John Carroll, le premier évêque catholique aux États-Unis, qui prescrivait une approche plus modérée de l'introduction du catholicisme à Boston ${ }^{43}$. Thayer, lui, s'en prenait aux prêtres de la région de Boston, débattait avec eux, puis publiait les comptes rendus de toutes ces controverses, à la manière des guerres de pamphlets entre puritains qui faisaient rage en Nouvelle-Angleterre depuis plus d'un siècle ${ }^{44}$. Il en vint même à adopter sa propre variante catholique de la croyance protestante commune qui veut que les événements séculiers annoncent l'histoire sacrée : «Peut-être, dis-je, celui qui élève et abat des empires, selon son bon vouloir, celui qui nomme et qui fait tout pour son Église, a permis à la surprenante Révolution à laquelle nous avons assisté d'éclater, puis de s'éteindre, afin d'accomplir quelques grands desseins et une bien plus joyeuse révolution dans l'ordre de la grâce $»^{45}$. Rétrospectivement, l'idée que la Révolution américaine fut un moyen par Dieu de promouvoir la diffusion du catholicisme romain semble bien étrange. Mais en 1787, soit à l'époque à laquelle le père John Thayer annonçait cette optimiste prophétie, c'est en fait une preuve irrésistible de la manière dont la réponse " américaine » aux transformations des relations avec la France fut extrêmement localisée; elle montre que les caractéristiques particulières de l'héritage commercial et culturel de Boston eurent une influence profonde sur la manière dont ses habitants s'adaptaient aux transformations qui touchaient le monde atlantique. À Boston, même les catholiques étaient puritains!

Les nouvelles relations entre la France et Boston, forgées pendant la Révolution américaine, offraient des débouchés commerciaux, pas simplement par la présence de la marine française à Boston, mais en permettant aux marchands de la ville de conduire et d'accroître un commerce légal avec la France. Avant la Révolution, le commerce était intensif mais illégal, et centré principalement sur la contrebande de sucre en provenance

(42) Abigail Adams à John Shaw, 18 janvier 1785, dans Richard Ryerson (éd.), Adams Family Correspondence, Cambridge, 199, p. $6: 63$.

(43) Jodziewicz, « American Catholic Apologetical ... », op. cit., p. 458-459.

(44) Controversy Between the Reverend John Thayer ... and the Reverend George Lesslie, Boston, 1793

(45) Conversion of the Reverend John Thayer, op. cit., p. 21. 
de la Caraïbe afin de contourner les taxes douanières restrictives imposées par le Parlement ${ }^{46}$. À la suite de la guerre d'Indépendance, la situation fut renversée et l'ancien négoce illégal fut ouvert aux navires américains. À Boston, l'exil de nombre des plus anciennes familles loyalistes permit à de nouveaux négociants, tels que les frères Perkins, James, Thomas et Samuel, de saisir ces nouveaux marchés.

Techniquement, les frères Perkins n'étaient pas des nouveaux venus. Ils étaient nés à Boston et leur famille était engagée dans le négoce depuis le XVII ${ }^{\mathrm{e}}$ siècle ${ }^{47}$. Mais, si leur grand-père et leur père furent raisonnablement prospères, ils n'avaient cependant jamais atteint le sommet de l'échelle des négociants de Boston, ni n'étaient devenus des personnalités de premier plan du monde politique ou social de la ville. Or, le plus tragique fut la disparition précoce en 1773 de James Perkins, le père des trois garçons, alors que son fils aîné n'avait que onze ans. Grâce à la boutique de leur mère, le foyer possédait un petit revenu, mais la fortune de la famille devait en souffrir. Les trois fils entamèrent leur apprentissage dans des maisons commerciales de Boston, plutôt que de poursuivre leurs études à Harvard, comme leurs talents et leur ascendance les y auraient autorisés, si leur père avait vécu. À l'âge adulte, au milieu des années 1780 , les garçons cherchèrent à fonder leur propre entreprise commerciale et furent attirés par la prospère colonie de SaintDomingue, accessible grâce à la nouvelle alliance avec la France ${ }^{48}$.

En 1783, James Perkins, le fils aîné, rejoignit une firme de courtiers de Boston déjà établie au Cap-Français. Il y apprit rapidement les affaires et devait bientôt se mettre à son compte. En 1785, son frère cadet, Thomas Handasyd Perkins, le rejoignit à Saint-Domingue et ensemble, associés à un troisième partenaire, ils fondèrent une affaire de négoce au Cap-Français. Quelques années plus tard, ils furent rejoints par leur plus jeune frère. Ils achetaient des marchandises importées au Cap par des concurrents et les revendaient à des planteurs locaux ${ }^{49}$. Évidemment,

(46) John J. McCusker, Rum and the American Revolution, New York, 1989; John W. TyLeR, Smugglers and Patriots : Boston Merchants and the Advent of the American Revolution, Boston, 1986.

(47) Robert Dalzell, Enterprising Elite: The Boston Associates and the World They Made, Cambridge, Harvard University Press, 1987.

(48) « Memoir of James Perkins », Massachusetts Historical Society, Proceedings, vol. 1 (1795-1835), p. 353-368. Voir aussi Thomas G. CARY, Memoir of Thomas Handasyd Perkins, Containing Extracts from his Diaries and Letters, Boston, 1856; Carl SEABurg et Stanley Paterson, Merchant Prince of Boston : Colonel T. H. Perkins, 1764-1854, Cambridge, 1971.

(49) Seaburg et Paterson, ibid., p. 33-43; « Memoir of James Perkins », op. cit., p. $353-$ 357. 
une partie importante de ces affaires concernait la traite des noirs ou leur ravitaillement afin de maintenir le système esclavagiste sur l'île. Au milieu des années 1780, la population servile de Saint-Domingue était comprise autour d'un demi million d'esclaves africains ou de descendants d'esclaves africains et était dirigée par une population blanche d'à peu près 30000 individus. Lors de la décennie pendant laquelle les frères Perkins se trouvaient sur l'île, la traite était à son apogée avec une importation annuelle d'esclaves comprise entre 30 et 40000 hommes, représentant un tiers de la traite vers l'Amérique entre 1783 et 1791 . Cette importation massive de main-d'œuvre était égale aux énormes exportations sortant de l'île. Saint-Domingue produisait alors $40 \%$ du sucre et $60 \%$ du café consommé en Europe ${ }^{50}$. Le Cap-Français représentait l'entrepôt commercial de ce commerce et les bénéfices acquis alors par la Maison Perkins furent le fondement des extraordinaires richesses qu'elle allait bientôt acquérir.

Même si chaque génération de la famille Perkins possédait des esclaves depuis le $\mathrm{XVII}^{\mathrm{e}}$ siècle, la traite n'avait jamais joué un rôle prépondérant dans leurs affaires, comme dans celles de la ville de Boston. Traditionnellement, les négociants bostoniens avaient obtenu de larges profits en subvenant à l'économie de plantation des Antilles, mais la demande limitée de main-d'œuvre au sein de la ville n'y avait jamais favorisé une traite importante. Mais ceci ne semble pas avoir ralenti l'ardeur des frères Perkins au Cap-Français tout comme le fait que l'État du Massachusetts ait aboli l'esclavage et la traite au début des années 1780. En tant que courtiers, l'achat et la revente d'esclaves importés constituaient une part régulière de leurs affaires au Cap-Français, tout comme le commerce de la farine, des poissons séchés et l'importation de chevaux qui permettaient aux plantations de fonctionner. Le café et le sucre qu'ils obtenaient en retour permettaient aux Perkins d'étendre leurs opérations commerciales à travers l'Atlantique, et bientôt tout autour de la planète ${ }^{51}$.

La contrebande jouait un rôle essentiel dans leurs affaires. Dans l'historiographie de la Révolution américaine, la contrebande active des marchands américains est souvent appréciée comme un acte patriotique et

(50) C. L. R. James, The Black Jacobins: Toussaint Louverture and the San Domingo Revolution, New York, 1963; Laurent Dubors, Avengers of the New World: The Story of the Haitian Revolution, Cambridge, 2004; David Geggus, "Saint-Domingue on the Eve of the Haitian Revolution », dans David Geggus et Norman Fiering, The World of the Haitian Revolution, Bloomington, 2009, p. 3-20.

(51) Seaburg et Paterson, Merchant Prince of Boston, op. cit., p. 144-145. 
de défiance envers les règles restrictives du Parlement ou encore comme un moyen de revendiquer une liberté commerciale et politique ${ }^{52}$. La faiblesse de ces arguments est clairement démontrée par les activités de la Maison Perkins et de bien d'autres à son image. La contrebande était simplement une activité économique, un pur acte d'intérêt commercial personnel, indifférent au viol des règles et à ceux qui les avaient édictées. En tant que dirigeants d'une petite ville-État opérant dans un monde contrôlé par d'immenses empires, les marchands de Boston considéraient la contrebande comme un moyen de maintenir une marge de concurrence et continuèrent à la pratiquer vigoureusement après la Révolution américaine et l'Indépendance des États-Unis.

La correspondance des frères Perkins contient des instructions détaillées adressées à leurs clients, leur expliquant comment éviter les frais de douane imposés par la France, l'Espagne, la Grande-Bretagne, la Chine, les États-Unis ou même l'État du Massachusetts. Ainsi une lettre de 1786 envoyée de Saint-Domingue à des clients précise : « J. P. sera à Boston fin septembre et il vous communiquera les moyens d'importer de la farine dans ce port et de récupérer les marchandises de contrebande $»^{53}$. Samuel Breck le jeune qui rejoignit l'affaire commerciale de son père en 1787 à Boston, à la suite de son séjour en France, connut, lui aussi, une expérience semblable. Il indique ainsi que les décrets portant sur les droits de douane du Massachusetts (qui avaient pris effet avant la ratification de la Constitution des États-Unis en 1789) étaient considérés avec beaucoup de réserves par les marchands de Boston eux-mêmes :

« Dès qu'un vaisseau arrivait, la moitié de la cargaison était hissée dans les parties supérieures de l'entrepôt, tandis que l'autre moitié pénétrait dans les bureaux des douanes ce qui fait que nous étions initiés aux secrets de la contrebande. Et demander une centaine de guinées à des jeunes gentlemen éduqués dans des principes honorables afin de leur enseigner ces basses fraudes et ce commerce déshonorant montre bien que notre époque marche sur la tête. Cependant, ceux qui jusqu'alors (et encore maintenant, du moins je l'espère) méprisaient ruses et tromperies en furent nommés les principaux agents. La seule excuse était le caractère universel de cette coutume $»^{54}$.

(52) Tyler, Smugglers and Patriots, op. cit.

(53) Seaburg et Paterson, Merchant Prince of Boston, op. cit. p. 41.

(54) Recollections of Samuel Breck, op. cit., p. 93. 
Les profits et les marchandises obtenus par les frères Perkins à Saint-Domingue ont contribué à former la base de leur prochaine et très lucrative entreprise. En 1788, le frère cadet, Thomas Handasyd Perkins, quitta le Cap-Français pour Boston afin de préparer leur prochain projet, le début de relations commerciales avec la Chine. Il affréta un cargo rempli de produits locaux, mais aussi de la Nouvelle-Angleterre (du beurre, du rhum, de la morue, des bougies à base de blanc de baleine) et des marchandises acquises à Saint-Domingue, comme une centaine de tonnes de barres de fer de Russie, que la Maison Perkins avait obtenue en développant la vente, jusqu'alors inédite, du sucre des Antilles à SaintPétersbourg ${ }^{55}$. L'Astrea partit pour la Chine en février 1789 et cette cargaison permit le début d'une nouvelle ère pour le commerce mondial de Boston. À Whampoa, l'ancien comptoir d'échanges sur la « rivière des perles » en aval de Canton, Thomas Handasyd Perkins constata l'intérêt soudain de la Chine pour les peaux de phoques et de loutres déchargées par un autre navire américain en provenance de l'Orégon sur la côte nord-américaine, ce qui, d'ailleurs nécessitait aussi des pratiques de contrebande afin d'éviter les agents de douane chinois! À partir de cet instant, il réorganisa les priorités commerciales de l'entreprise familiale et, en raison de leur immense succès, la communauté des marchands de Boston fit de même et en vint à dominer le marché américain du nordouest du Pacifique et de la Chine pendant plusieurs décennies ${ }^{56}$.

Ce sont les bénéfices tirés du commerce de Saint-Domingue qui permirent cet important développement, James et Samuel Perkins continuant dans cette voie, poursuivant leur commerce après l'annonce du décret de l'Assemblée constituante française du 15 mai 1791, qui accordait l'égalité aux «hommes de couleurs et Noirs libres », et même à la suite des violences qui l'accompagnèrent au Cap. À l'automne 1791, les révoltes des esclaves éclataient dans les plantations situées dans les collines se trouvant au-dessus du Cap-Français. James Perkins et sa famille étaient justement en visite dans une de ces plantations lorsque l'on sonna l'alarme et, après un voyage atroce, ils purent finalement s'échapper dans un attelage et rejoindre la ville ${ }^{57}$. Et malgré l'incendie qui devait atteindre Port-au-Prince et les champs de canne à sucre en flammes au-dessus de la

(55) Kalevi Ahonen, From Sugar Triangle to Cotton Triangle : Trade and Shipping between America and Baltic Russia, 1783-1860, University de Jyvaskyla, Finlande, 2005, p. 362-376.

(56) Memoir of Thomas Handasyd Perkins, op. cit., p. 42-44; SEABUrg et Paterson, Merchant Prince of Boston, op. cit., p. 43-60.

(57) « Memoir of James Perkins », op. cit., p. 53-68. 
ville, les affaires continuaient comme d'habitude au Cap-Français. James Perkins devint le commandant des Américains blancs engagés dans la défense de la ville, tandis que se poursuivait l'arrivée d'esclaves en provenance d'Afrique, avec l'espoir de les vendre une fois l'ordre revenu dans les plantations, ou bien, s'il le fallait, à la Havane ou en Caroline du Sud. Comme beaucoup d'Américains, les frères Perkins considéraient le début de la Révolution en France comme un événement heureux, un signe montrant qu'un autre pays se tournait, lui aussi, vers le modèle républicain et l'autonomie. Toutefois, ils étaient incapables d'apprécier les révoltes des esclaves de Saint-Domingue comme faisant partie du même processus, ou encore d'imaginer un gouvernement autonome et indépendant dirigé par la majorité africaine de l'île. Ils continuèrent donc à travailler au Cap jusqu'à ce que la ville, en 1793, tombe aux mains des révoltés, Samuel Perkins décidant alors de retourner à Boston, abandonnant des sommes impayées que lui devaient les planteurs de Saint-Domingue ${ }^{58}$.

Pourtant, la violence de la Révolution à Haïti ne fut pas un frein au soutien de la Maison Perkins à la France et à sa Révolution. En janvier 1793, Russel Sturgis, époux d'Elizabeth, l'une des sœurs Perkins, et membre associé de la firme, organisa une «Fête Civique en commémoration de la glorieuse résistance et des brillants succès des citoyenssoldats de la Liberté en France $\aleph^{59}$ après que les nouvelles de la victoire française de Valmy sur le duc de Brunswick (les troupes du Brunswick et de la Hesse avaient menacé Boston lors de la Révolution américaine) et de l'abolition de la monarchie furent parvenues sur le sol américain. Ces événements, mêlés aux massacres de Septembre, ainsi que les débuts d'une déchristianisation légale de l'État français, semblaient indiquer aux Bostoniens que les deux piliers de la tyrannie de leurs anciens « ennemis gaulois », la monarchie absolue et le catholicisme romain, étaient en train de s'effondrer ${ }^{60}$. La fête eut lieu à Faneuil Hall, le célèbre marché public que le marchand huguenot Peter Faneuil dans les années 1740 avait donné à la municipalité de Boston. Un bœuf entier fut rôti pour l'occasion afin de nourrir la foule, le tout accompagné de feux d'artifices et de joie, souvenir des célébrations antipapistes anciennes, mais qui à présent honoraient les Français ${ }^{61}$.

(58) Seaburg et Paterson, Merchant Prince of Boston, op. cit., p. 72-88.

(59) The Columbian Centinal, Vol. 18, no 38, 19 janvier 1793, p. 3 ; Chandler RobBins, An Address, Delivered at Plymouth, on the $24^{\text {th }}$ day of January, 1793, ... to celebrate the victories of the French Republic, Boston, 1793.

(60) JoDKIEWICZ, op. cit., p. 458-459.

(61) Elkins et McKitrick, The Age of Federalism, op. cit., p. 310. 
Lors du début des hostilités entre la Grande-Bretagne et la France, George Washington, le président des États-Unis, choisit la neutralité. Cette position fut vécue pour les frères Perkins comme une nouvelle chance d'étendre la prospérité du commerce de Boston dans l'Atlantique en développant un commerce sans intermédiaire. La guerre signifiait des contrats pour l'approvisionnement des troupes et le caractère destructif des guerres de l'époque révolutionnaire en France créait des disettes. Le pain était très demandé et des émeutes de la faim avaient éclaté en février 1793 à Paris, peu de temps après la déclaration de guerre contre la Grande-Bretagne. En juin, James Perkins quitta Boston pour les Antilles (s'arrêtant en Martinique, Saint-Domingue étant en flammes), puis continua son voyage vers la France où un profit de $200 \%$ pouvait être espéré sur la vente de grains venus des États-Unis ${ }^{62}$. James Perkins fut de retour à Boston en janvier 1794, et malheureusement, nous ne possédons aucune trace de son expérience et de ses observations pendant cette période passée sous la Terreur. Toutefois, son frère, Thomas Handasyd Perkins, à l'automne de la même année, fit le même voyage, empruntant les mêmes routes, et passa l'année 1795 à voyager en France, poursuivant ses affaires commerciales à Paris tout en tenant un journal détaillant ses impressions et les événements dont il était le témoin.

Et, curieusement, on n'y trouve pas les réactions stéréotypées auxquelles on pourrait s'attendre de la part d'un marchand de Boston, ou plutôt, de ce que l'historiographie dominante nous a invité à penser. Perkins arrive à Bordeaux dans les premiers jours du mois de février 1795. Il rejoint Paris par l'intérieur des terres : « Nous passâmes si près du théâtre des affrontements de la guerre de Vendée que nous pouvions pratiquement entendre le son du canon $»^{63}$. À son arrivée à Paris, la disette est tragique : " Il y avait une grande pénurie de pain depuis l'hiver et le printemps. La raison provenait en partie du fait que les fermiers avaient vu leurs charrues être transformées en armes, en partie en raison des pertes dues à la guerre et en partie en raison de la défiance qui entourait les assignats », leur dépréciation étant un problème connu par les Américains qui se rappelaient leur fragile état financier lors de leur propre révolution ${ }^{64}$. Perkins arriva lors des derniers actes de violence qui suivirent la chute des Montagnards. Il assista même à l'exécution de Fouquier-Tinville et de quinze juges et jurés du Tribunal révolutionnaire. En utilisant sa montre

(62) Seaburg et Paterson, Merchant Prince of Boston, op. cit. p. 106-108.

(63) Memoirs of Thomas Handasyd Perkins, op. cit., p. 54.

(64) Ibid, p. 55 
placée dans sa poche, il estima que « de l'instant où les prisonniers sont descendus de la charrette au moment où leurs têtes détachées de leurs corps ont été placées dans de longs paniers, quatorze minutes s'étaient écoulées ${ }^{65} »$. Bien entendu, il fut affecté par la violence et inquiet qu'une exposition régulière à de telles scènes sanglantes pût endurcir le peuple français et le rendre insensible à la détresse humaine ${ }^{66}$. Il participa à l'entreprise clandestine qui consista à essayer d'enlever le fils du héros français de la Révolution américaine, le marquis de La Fayette, alors en exil, afin qu'il échappe à son triste sort ${ }^{67}$. En mars 1795 , il assista aux procès de Barère, Collot d'Herbois et Billaud-Varenne, et il dîna avec les conventionnels qui souhaitaient les punir sans toutefois les imiter :

« Ils n'hésitaient pas à avancer que ces procès prenaient la forme d'un jugement entre le Jacobinisme et ses excès et le règne de la raison et de la modération. Que Dieu fasse triompher ces derniers. Ils souhaitent guillotiner leurs chefs, qui sont à présent en procès, tout ne poursuivant pas leurs complices. Si les modérés devaient poursuivre tous ceux ayant participé au système actuellement en procès, cela ne ferait que détruire un système de terreur par un autre $»^{68}$.

Lorsque la Convention rendit son verdict et condamna Barère et ses alliés à la déportation plutôt qu'à être exécutés, Perkins exprima sa satisfaction :

« Je pense qu'il s'agit d'une décision très politique de la part de l'Assemblée de ne pas avoir répandu le sang de ceux qui pourtant le méritaient, comme Barère et ses collègues unis dans l'injustice. Il aurait été dangereux de réactiver la guillotine $»^{69}$.

Malgré le sang versé et les injustices de la Terreur, Perkins ne perdit jamais espoir et croyait encore aux mérites de la France et de la Révolution française. Il appréciait les arts, l'architecture et la culture française. Il se rendit à l'Opéra, visita Ermenonville et le château de Versailles. En témoin attentif de la vie urbaine, du commerce et du négoce, il se

(65) Ibid., p. 112-113.

(66) Ibid.,p. 113-114. Voir également, Rachel Hope CLEVEs, The Reign of Terror in America : Visions of Violence from Anti-Jacobinism to Antislavery, Cambridge, 2009.

(67) Seaburg et Paterson, Merchant Prince of Boston, op. cit., p. 110-111; Memoirs of Thomas Handasyd Perkins, op. cit., p. 57-59.

(68) Ibid., p. 77.

(69) Ibid., p. 87. 
rendit dans plusieurs centres manufacturiers, observant leurs méthodes pour fabriquer des poteries, de la porcelaine, du fer ou des draps. Un de ses amis, compagnon de voyage, Joseph Russell, négociant et bostonien comme lui, utilisa ses bénéfices commerciaux afin d'acheter un château situé dans la campagne d'île-de-France. À Paris, Perkins fut stupéfait par l'esprit public des gardes nationaux, des dizaines de milliers de volontaires se succédant quotidiennement afin de garantir la paix et prévenir un retour des Jacobins lors des procès du printemps 1795. Il observa le défilé des troupes françaises devant les Tuileries et releva la beauté des « canonniers» : " la République leur doit ses plus belles victoires. Prions que chacun d'entre nous soit un canonnier lorsque opposé aux tyrans et que chaque canonnier soit une armée à lui tout seul $»^{70}$ ! À la suite de sa visite de l'Hôtel des Invalides, il explique que « lorsque l'on contemple les causes qui ont amené à autant d'injustices envers ces pauvres gens [...] et que l'on les identifie comme provenant de l'ambition des rois ou des tyrans, selon le nom que l'on leur donne, on se prend à espérer que le monde s'en débarrasse et adopte la forme du gouvernement républicain $»^{71}$.

Perkins en arrive à des conclusions semblables lorsqu'il se rendit à l'endroit où « la Bastille se tenait [...] Et lorsque se rend compte de la misère contenue dans ce lieu, le sang vous glace les veines et on s'emporte rageusement contre les tyrans, où qu'ils se trouvent et quels qu'ils soient. Malheureusement, on observe encore bien des Bastilles en France depuis que celle-ci fut abattue ${ }^{72}$. Toutefois, il estimait que les excès de la Révolution étaient à présent terminés. De même, il approuvait la décision de la commission nommée par la Convention de donner une nouvelle Constitution à la République française, précisant qu'elle «devrait permettre au pays, déchiré par des discordes généralisées, de retrouver un solide et durable gouvernement. Ses principes sont d'ailleurs très semblables à ceux sur lesquels notre propre gouvernement repose ». Perkins aurait cependant préféré un pouvoir exécutif confié à un seul homme et possédant un droit de veto limité plutôt qu'un Directoire de cinq membres, à la mode américaine. Toutefois, il voyait dans cette nouvelle forme de gouvernement un élément de stabilisation, vecteur d'ordre et de paix pour la République $^{73}$. Mais, le plus important, c'est que tout cela confirmait ses 
convictions au regard de ses attentes commerciales. Ainsi, à la suite d'un nouveau repas en compagnie de membres de la Convention, il pouvait écrire :

« Pelet nous questionna énormément à propos de notre commerce et il semblait anxieux de savoir quel serait l'état de nos relations avec la France aux lendemains de la guerre. Sur ce point, je n'ai aucun doute, nos relations seront fortes, ce qui d'ailleurs nous sera hautement profitable. Il me rapporta que les récoltes de blé en France ne couvrent jamais la demande $[\ldots]$ et que ce blé, au mieux, vaut douze livres tournois en espèce le quintal. Ceci ferait de bonnes cargaisons venues d'Amérique en temps de paix $»^{74}$.

En résumé, malgré ses observations intimes concernant les suites $\mathrm{du}$ « règne de la Terreur » et les turbulences de la vie politique française, Perkins semblait suffisamment confiant dans le succès de la République pour imaginer que le commerce de Boston puisse, à l'avenir, y jouer un rôle éminent.

Cette confiance inaltérable des frères Perkins, en cette fin de l'année 1795, dans les promesses commerciales et politiques de la République française, malgré les violences de la Terreur et le traité de Jay avec la Grande-Bretagne qui avaient, soi-disant, retourné les Fédéralistes de la Nouvelle-Angleterre contre la France, nous rappelle l'importance de s'attacher aux imprévus de l'histoire. Cette dimension doit être tout particulièrement prise en considération à l'époque des révolutions atlantiques, lorsque les extraordinaires turbulences des affaires publiques rendaient les prédictions sur l'avenir impossibles à tenir, même pour des marchands dont la fortune provenait de ce talent. Rétrospectivement, nous savons que les héritiers de Jefferson et les démocrates-républicains aux États-Unis ont continué à soutenir la France et à s'opposer à la GrandeBretagne pendant les premières décennies du $\mathrm{xIX}^{\mathrm{e}}$ siècle. Nous savons également que leurs opposants, les fédéralistes, dont un nombre important provenait de Boston et de la Nouvelle-Angleterre, s'opposeraient à la « Guerre de M. Madison » de 1812 contre la Grande-Bretagne. Mais la puissance narrative de l'histoire politique intérieure des États-Unis a trop souvent amené les historiens à placer trop précocement et sans nuance la Nouvelle-Angleterre et Boston dans le camp des anti-Français et des anti-révolutionnaires. 
Pour reprendre de nouveau les mots de Franklin, « les affaires du peuple d'Amérique ont trop souvent été considérées comme un tout ». La ville-État de Boston devait rester fidèle à elle-même au début de la République, ne s'intégrant jamais aisément aux États-Unis dans leur ensemble, et ouvrant la voie, en 1814, à un mouvement sécessionniste ${ }^{75}$. Les premières réactions de Boston à la Révolution française furent assez extraordinaires surtout si l'on considère son antipathie historique envers la France avant 1776. Il fallut donc attendre l'arrivée de Napoléon au pouvoir à la fin des années 1790, ainsi que le développement d'une lecture de la Révolution française comme le passage d'une anarchie démocratique à une tyrannie impériale, sans passer par une république autonome et raisonnable, pour que la Maison Perkins finisse par quitter la France.

Toutefois, il y avait aussi des fédéralistes à Boston qui avaient réagi dès le début des événements français à partir du prisme de la politique nationale américaine, des hommes comme Fisher Ames qui considéraient le parti de Jefferson comme un danger pour les États-Unis et qui s'opposaient à la Révolution simplement parce que Jefferson y adhéraii ${ }^{76}$. Mais, pour des hommes comme les frères Perkins, qui s'intéressaient plus à la fortune immédiate de leur ville qu'aux rebondissements de la politique nationale, et qui estimaient que la toute jeune République américaine faisait partie des nombreuses puissances commerciales rattachées à leur domaine de négoce, la République française et la ville de Boston pouvaient envisager de devenir des partenaires, jusqu'au moment où cette perspective ne serait plus envisageable.

Mark Peterson

Department of History University of California

Berkeley, California 94720-2550 mark-peterson@berkeley.edu

(75) James M. BAnner, Jr., To the Hartford Convention: The Federalists and the Origins of Party Politics in Massachusetts, 1789-1815, New York, 1970; Cleves, Reign of Terror, op. cit., p. 191-193.

(76) Linda K. Kerber, Federalists in Dissent: Imagery and Ideology in Jeffersonian America, Ithaca, 1970. 